\title{
ANÁLISIS DE LAS ESPECIFICACIONES DE MATERIALES UTILIZADOS PARA EL HORMIGÓN PRETENSADO A BAJAS TEMPERATURAS
}

\author{
Manuel Elices Calafat y Jaime Planas Roselló, Ingenieros de Caminos*
}

\section{INTRODUCCIÓN}

En la construcción de depósitos para contención de gases licuados refrigerados se incorporan dos tecnologías básicas: la clásica de estructura metálica, que hace uso de aleaciones especiales, y la del hormigón pretensado.

Las estructuras de estas caracteristicas, particularmente los depósitos de Gas Natural Licuado (GNL), deben cumplir requisitos muy estrictos de seguridad frente a acciones accidentales y se diseñan buscando doble seguridad, basada en el recipiente propiamente dicho y en un depósito externo de protección, que debe hacer frente a acciones exteriores y a eventuales fugas o roturas en el recipiente interno. El depósito interno puede ser metálico (Depósito de Huelva, y los primeros depósitos de Barcelona, por ejemplo), o de hormigón pretensado (último depósito de Barcelona). El depósito externo es, en general, de hormigón pretensado, aunque existe otro tipo de soluciones basadas en geomateriales (depósitos enterrados o protecciones con materiales sueltos).

Debido a la relativa rareza de este tipo de estructuras, que no favorece su estudio continuado, y a las dificultades que plantean la propia complejidad de los fenómenos que tienen lugar al disminuir la temperatura y el elevado coste de la investigación experimental en este campo, no existe en la actualidad una normativa universalmente aceptada para la caracterización y control de los materiales que forman parte de un depósito pretensado.

En este artículo se analizan las prescripciones desarrolladas por KELLOGG para ENAGAS con ocasión del proyecto y construcción del tanque de GNL de Huelva. Se tratan sucesivamente los materiales que integran una estructura de hormigón pretensado, en este orden: Hormigón, Armaduras Pasivas y Sistemas de Pretensado. Para cada material se exponen someramente las propiedades básicas, se presentan y discuten los aspectos esenciales de las Prescripciones KELLOGG y se proponen alternativas a los puntos más conflictivos.

\section{HORMIGÓN}

\subsection{Propiedades esenciales del hormigón en condiciones criogénicas}

En el intervalo de bajas temperaturas el hormigón presenta algunas propiedades especiales que deben ser tenidas en cuenta en el diseño e interpretación de resultados de ensayos de caracterización.

La primera es que el contenido de humedad del hormigón condiciona de forma notable todos los aspectos del comportamiento mecánico. Así como en el intervalo de uso ordinario puede prescindirse, a efectos prácticos, de la influencia de esta variable, no es posible hacerlo cuando se consideran temperaturas por debajo de los $-10 \mathrm{C}$ aproximadamente. Sólo como ejemplo diremos que el paso de seco a saturado produce variaciones del $200 \%$ en la resistencia a compresión a $-170 \mathrm{C}$. Variaciones más espectaculares, si cabe, del comportamiento se dan para los fenómenos de contracción y dilatación térmica y para la resistencia a ciclos hielo-deshielo.

La segunda propiedad es que las caracteristicas mecánicas mejoran a lo largo de un enfriamiento monótono. Esto ha sido establecido hace tiempo por diversos autores para la resistencia a compresión y a tracción y para la adherencia hormigón-armadura. Recientemente, ensayos realizados en nuestro Departamento han mostrado que la tenacidad de fractura del hormigón saturado se multiplica por tres al enfriar hasta $-170 \mathrm{C}$ y que la fragilidad estructural es del orden de la cuarta parte de la disponible a temperatura ambiente. La consecuencia fundamental es que mientras en el proyecto se utilicen como valores de cálculo los valores característicos a temperatura ambiente, los aspectos resistentes no deben ser preocupantes para operación continuada a baja temperatura.

Sin embargo, si se considera la posibilidad de ciclos de enfriamiento-calentamiento debe tenerse en cuenta que pueden producirse pérdidas elevadas de resis-

*Catedráticos del Departamento de Ciencia de Materiales. E.T.S. de INGENIEROS DE CAMINOS (Universidad Politécnica de Madrid). 
tencia y de rigidez cuando el contenido de humedad es elevado. La importancia de la humedad en este com. portamiento se pone de manifiesto en la Fig. 1, en la que se representa la resistencia residual de probetas de hormigón sometidas a ciclos térmicos en función del número de ciclos, para dos condiciones de conservación: saturación y $65 \%$ de humedad relativa. Nótese que la pérdida de resistencia para 12 ciclos es del orden de 5 veces mayor para la probeta saturada que para la probeta seca.

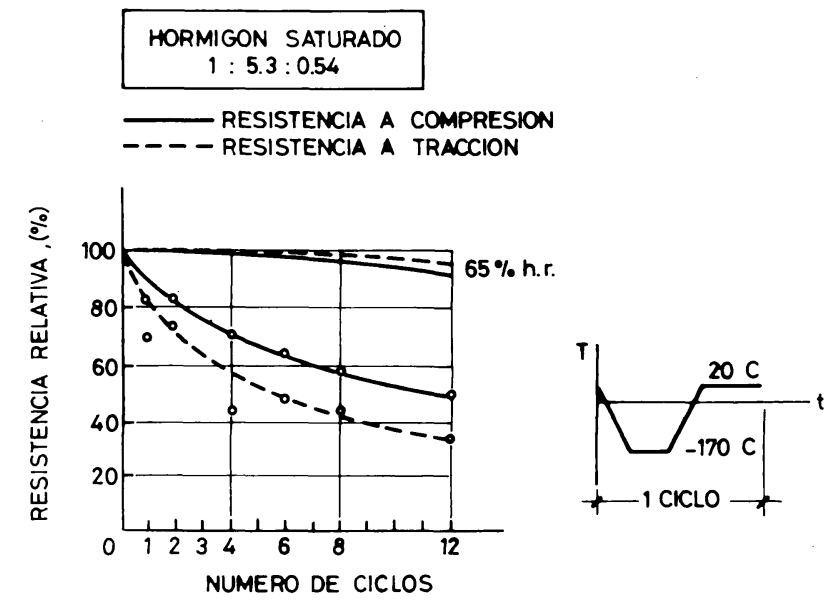

Fig. 1

El último aspecto a resaltar corresponde a la influencia de la carga durante el proceso de enfriamiento. En el intervalo habitual de temperaturas, en tanto las cargas no sobrepasan un cierto nivel, las cargas térmicas se calculan suponiendo válido el principio de superposición para deformaciones de origen mecánico y térmico. Para un proceso de enfriamiento por debajo de los $-10 \mathrm{C}$ tal superposición ya no es posible para hormigones con un contenido de humedad apreciable. La Fig. 2 muestra, en efecto, que las curvas de deforma-

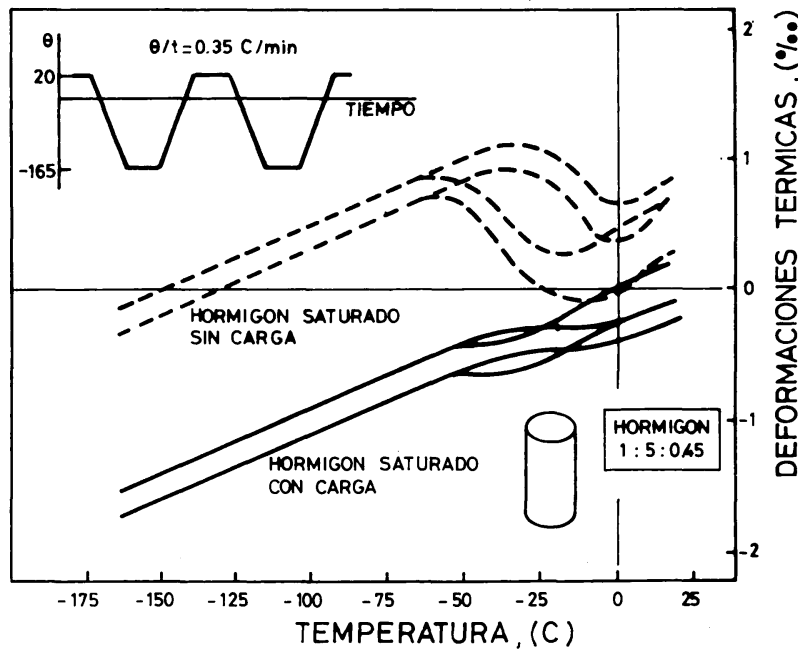

Fig. 2 ción térmica frente a la temperatura son completamente distintas para probetas sin carga y probetas con una carga uniaxil constante que simula las fuerzas de pretensado. La consecuencia obvia es que en estructuras pretensadas, que se enfrían bajo carga aproximadamente constante, los resultados de ensayos de dilatación térmica realizados bajo carga nula son del todo irrelevantes para proyecto. Debe notarse, de nuevo, que la influencia de la humedad es fundamental, y que para probetas desecadas desaparece el efecto no lineal anteriormente expuesto.

\subsection{Sobre las prescripciones KELLOGG}

Las especificaciones KELLOGG establecen dos grupos bien diferenciados de ensayos: el grupo de ensayos de recepción y control y el grupo de ensayos de caracterización.

Los ensayos del primer grupo son idénticos para recepción y control, modificándose solamente los tamaños de muestra. Estos ensayos son los únicos que incluyen condiciones de aceptación. La ejecución de los ensayos de caracterización es preceptiva y debe realizarse en paralelo con los de recepción, pero sus resultados son informativos y no llevan aparejados criterios de aceptación o rechazo.

\section{Ensayos de recepción y control}

Los ensayos de caracterización y control son, en esencia, dos ensayos de heladicidad. En el primero, que llamamos de choque térmico, se mide a temperatura ambiente el módulo de elasticidad dinámico de las probetas antes y después de un ciclo de enfriamiento brusco por inmersión en nitrógeno líquido $(-196 \mathrm{C})$ y posteriormente se determina su resistencia a compresión. En el segundo se efectúan las mismas determinaciones, pero sometiendo las probetas a 20 ciclos térmicos entre $+5 \mathrm{y}-20 \mathrm{C}$.

Sin entrar en detalles de procedimiento, la condición de aceptación es que la resistencia a compresión y el módulo de elasticidad dinámico no disminuyan, después de los ciclos térmicos, en más de un $20 \%$.

Como se ha indicado en el apartado anterior, el contenido de humedad influye decisivamente en el efecto de los ciclos térmicos y por tanto las condiciones de conservación son esenciales. En las especificaciones sólo se requiere que las probetas, después del período habitual de curado, se conserven a una humedad relativa no inferior al $90 \%$. En el apartado siguiente se expondrán razones que indican que el margen para las condiciones de conservación es excesivo y se propondrá una solución alternativa. 
Puesto que las determinaciones se realizan a temperatura ambiente, estos ensayos son de fácil realización. La inmersión en nitrógeno líquido es trivial, y existen equipos comerciales para la realización automática de ciclos entre +5 y $-25 \mathrm{C}$. La única precaución esencial es evitar las modificaciones de humedad de las probetas durante su manejo y ensayo.

Si la conservación se efectúa en las proximidades del $90 \%$ de humedad relativa no existen dificultades especiales en conseguir un hormigón que supere los ensayos si se toman las precauciones habituales al efectuar la dosificación: utilización de árido poco absorbente, utilización de relaciones agua/cemento del orden de 0,45 o inferiores y utilización de aireantes en proporción adecuada.

\section{Ensayos de Caracterización}

Las especificaciones KELLOGG consideran 5 tipos de ensayos de caracterización:

1. Ensayos de tracción indirecta por el método brasileño.

2. Ensayos de compresión uniaxil con registro de curva tensión-deformación.

3. Ensayos de tracción directa.

4. Ensayos de conductividad térmica.

5. Ensayos de coeficiente de dilatación térmica.

Las especificaciones no incluyen procedimientos detallados de ensayo y se limitan a establecer que el Contratista debe desarrollar dichos procedimientos y someterlos a la aprobación de la Propiedad. Fija, sin embargo, algunas de sus características básicas. Estipula que deben realizarse los ensayos anteriores a cinco temperaturas distintas entre 30 y $-170 \mathrm{C}$ y establece que se harán para probetas con dos humedades distintas, conseguidas mediante un acondicionamiento adecuado.

KELLOGG define unos acondicionamientos "ideales" para los cuales las probetas tendrían, en el momento del ensayo, contenidos de humedad iguales, respectivamente, al máximo y mínimo contenido de humedad que pueden esperarse en la estructura, teniendo en cuenta las condiciones atmosféricas reinantes en el punto de construcción. Sin embargo, indica que a falta de información exhaustiva, los máximos y mínimos contenidos de humedad pueden conseguirse manteniendo las probetas a humedades relativas del $90 \mathrm{y}$ del $50 \%$, respectivamente, durante 28 dias adicionales a los 28 de conservación normal a humedad relativa no inferior al $90 \%$.

Por lo que se refiere a los ensayos propiamente dichos, cabe recalcar que cada uno de ellos requiere montajes experimentales distintos y relativamente comple- jos. Todos ellos necesitan un control estricto de la temperatura (el de conductividad térmica, en particular, requiere una excelente estabilidad en la temperatura impuesta) y, si exceptuamos los ensayos brasileños y los de conductividad, requieren medidas de deformaciones a bajas temperaturas. Son, en resumen, ensayos muy fuera de lo corriente que requieren equipos muy especializados que no se encuentran ordinariamente en un laboratorio de ensayo de materiales de ingeniería.

Sin embargo, si se hace abstracción de las dificultades de ejecución de los ensayos, se ve que no presentan dificultades conceptuales especiales en cuanto a su posible interpretación. Excepción a esta regla son los ensayos de tracción directa y de dilatación térmica. Estos dos tipos de ensayo, por sus características peculiares, se analizan con mayor profundidad a continuación.

\section{a) Ensayos de tracción directa}

Las especificaciones KELLOGG admiten dos posibles metodologías de ensayo. En la primera se realizan ensayos "ordinarios" de tracción simple a diferentes temperaturas, con medida de deformaciones, de forma que en cada temperatura de ensayo se obtienen el módulo de elasticidad en tracción y la resistencia a tracción. En la segunda se realizan ensayos de "deformación im. pedida", en los cuales la probeta, inicialmente a temperatura ambiente, se enfría de forma continua manteniendo nula su deformación de manera que la carga aumenta por efecto térmico hasta producirse la rotura. De este segundo tipo de ensayos se obtiene la resistencia a tracción y una curva temperatura-carga de la que puede obtenerse el producto del módulo de deformación por el coeficiente de dilatación térmica.

Las especificaciones KELLOGG recomiendan la utilización del segundo método de ensayo. Sin embargo, debe tenerse en cuenta que la información suministrada por este segundo tipo de ensayos es muy limitada. En primer lugar, es de notar que suministra información solamente en el entorno de temperatura ambiente, ya que, de un cálculo elemental, resulta que la variación de temperatura que lleva a la rotura es del orden de la deformación en el pico de carga esperable en tracción (100-200 $\mu \mathrm{m} / \mathrm{m}$, típico) dividida por el coeficiente de dilatación térmica $(10 \mu \mathrm{m} / \mathrm{mC}$, típico) lo que da valores de $10-20 \mathrm{C}$, con lo que la rotura se produce a temperatura superior a $0 \mathrm{C}$. En segundo lugar, los resultados son de difícil interpretación debido a que se trata de un proceso a carga y temperatura variable, con lo que los resultados "mezclan" propiedades térmicas y propiedades mecánicas que no pueden separarse a menos que se suponga que el proceso es lineal. 


\section{b) Ensayos de dilatación térmica}

Este es uno de los puntos en que las especificaciones KELLOGG resultan más ambiguas. Se limita a decir que debe obtenerse el coeficiente de dilatación térmica a diferentes temperaturas. No especifica si se trata del coeficiente tangente $o$ del coeficiente secante y esto tiene importancia práctica, a menos que se trate de un material con coeficiente de dilatación constante y no es éste el caso. En los ensayos realizados por nosotros el problema se obvia obteniendo la curva completa deformación térmica-temperatura, de la cual pueden extraerse tanto valores tangentes como secantes.

En cualquier caso, el problema más grave es el que surge del efecto de la carga durante el proceso de enfriamiento, mencionado en el apartado 2.2., de manera que para obtener valores razonables de las contracciones térmicas que va a experimentar el hormigón en la estructura pretensada, sería necesario efectuar ensayos de probetas sometidas a carga constante de compresión, con niveles de carga similares a los que el hormigón va a tener en obra.

\subsection{Propuesta de modificación de especificaciones}

Los problemas esenciales que se han revelado en la exposición anterior son fundamentalmente tres:

1. Definición precisa de las humedades de ensayo.

2. Definición de las condiciones de los ensayos de tracción.

3. La dilatometría sobre probetas descargadas es irrelevante.

El primer problema surge de la influencia del contenido de humedad del hormigón en los resultados de los ensayos y de la fuerte dependencia del contenido de humedad respecto de las condiciones de conservación. Los resultados de Rostasy detallados en Elices et al, (1982) indican que el contenido específico de agua para probetas conservadas al $100 \%$ de humedad relativa es de $65 \mathrm{~g} / \mathrm{kg}$ en tanto que las conservadas al $90 \%$ de humedad relativa retienen solamente $49 \mathrm{~g} / \mathrm{kg}$. Considerando las primeras como saturadas, el grado de saturación de las segundas es del $75 \%$, lo que implica que queda cámara de aire suficiente para acomodar la expansión del agua al congelarse. Se tiene, en consecuencia, que los resultados de ensayos de heladicidad y dilatometría llevarán a resultados francamente distintos si la conservación se efectúa al $90 \%$ al $100 \%$ de humedad relativa. Por lo tanto, la condición de humedad relativa igual o superior al $90 \%$ es demasiado amplia, ya que es posible que los ensayos de un laboratorio que conserve las probetas al $91 \%$ den resultados aceptables, en tanto que conservándolas al $100 \%$ de humedad relativa, el mismo hormigón deba ser rechazado.

(C) Consejo Superior de Investigaciones Científicas

Licencia Creative Commons 3.0 España (by-nc)
Por otra parte, limitar el intervalo de condiciones de conservación, que es una posible solución, lleva a dos problemas: Primero, definir el intervalo de validez, que es bastante arbitrario, y segundo, y más importante, definir el nivel medio que puede dar lugar a condiciones demasiado restrictivas o demasiado permisivas. La solución más razonable puede estar en una conservación sellada. Esta solución se basa en los cálculos de Rostasy incluidos en Elices et al, (1981) que indican que la mayor parte de la sección de un muro de espesor típico $(40-50 \mathrm{~cm})$ no experimenta cambios de humedad con el ambiente a lo largo de toda su vida útil, debido a la lentitud de los procesos de difusión hídrica.

Proponemos, en consecuencia, que todos los ensayos se realicen sobre probetas que se conserven selladas. Por supuesto debe garantizarse la eficiencia del sellado, lo que puede hacerse imponiendo una pérdida máxima de peso en 28 dias. Un valor razonable para la pérdida máxima de peso es de $0,3 \mathrm{~g} / \mathrm{kg}$, que conduce a variaciones en el grado de saturación del orden del $5 \%$.

En relación a los ensayos de tracción directa, de acuerdo con lo anteriormente expuesto, el primer método de ensayo suministra mucha mayor información que el segundo, y, sobre todo, la información es mucho más fácil de interpretar que en el segundo método, que es el recomendado por KELLOGG. Recomendamos, por tanto, que se utilicen ensayos de tracción directa convencionales a diferentes temperaturas.

Por lo que concierne a los ensayos de dilatación térmica, resulta evidente de lo anteriormente expuesto que los ensayos bajo carga nula son poco representativos para predecir el comportamiento del hormigón en estructuras pretensadas, por lo que recomendamos que la medida de dilataciones se efectúe sobre probetas sometidas a tensión uniaxil constante. Con más precisión, basándonos en los resultados obtenidos en el laboratorio (J. Planas et al, 1984, M. Elices et al, 1986 y $H$. Corres et al, 1986), los ensayos deberían realizarse con probetas sometidas a las cargas medias que se prevean en el proyecto y con una humedad lo más cercana a la previsible.

\section{ACERO DE PRETENSADO}

\subsection{Comportamiento en condiciones criogénicas}

En términos generales, las propiedades a $-170 \mathrm{C}$ del acero eutectoide trefilado, comparadas con sus valores a temperatura ambiente, experimentan la siguiente evolución (M. Elices et al, 1982):

- Módulo de elasticidad; aumenta ligeramente ( $\cong 12 \%$ ). - Límite elástico: aumenta ligeramente ( $\cong 15 \%$ ). 
- Tensión de rotura: aumenta ligeramente ( $\cong 15 \%$ ).

- Deformación bajo tensión máxima: se mantiene o disminuye ligeramente.

Mención aparte merece el problema de la fragilización de este material a bajas temperaturas. En primer lugar, la medida de la evolución a la tenacidad de fractura del material virgen (enfriado sin carga y posteriormente llevado a rotura) efectuadas en nuestro departamento ( $M$. Elices, 1985) muestran que en el intervalo de temperaturas $(-196,20)$ no existe una temperatura de transición propiamente dicha, sino una disminución suave de la tenacidad, con una reducción máxima del orden del $50 \%$. En segundo lugar, la forma de utilización estructural del material disminuye enormemente los riesgos de rotura frágil porque se carga a temperatura ambiente a una carga superior a la de servicio antes de ser enfriado y posteriormente se enfría estando traccionado. La puesta en tensión constituye en sí misma una prueba de carga que eliminaría, caso de existir, todos los elementos con defectos superiores al crítico y la plastificación local que se produce en el entorno de posibles defectos subcríticos eleva sustancialmente la tenacidad efectiva disponible a baja temperatura respecto de la medida para el material virgen. Se trata de un efecto similar al "warm prestressing" (véase, por ejemplo, M. Elices, 1985).

Si a estas consideraciones añadimos el buen comportamiento observado en conjuntos tendón-anclaje en condiciones criogénicas (J. Planas et al, 1985) en las que las solicitaciones pueden considerarse extremas y el hecho ya mencionado de que la rotura accidental de un alambre no supone el colapso estructural frágil, podemos concluir que, en principio, el acero eutectoide trefilado térmicamente tratado presenta excelentes caracteristicas para su uso a bajas temperaturas.

\subsection{Sobre las prescripciones KELLOGG}

La correspondiente especificación KELLOGG establece un único criterio de aceptación, a saber: que la deformación unitaria bajo carga máxima sea superior al $2 \%$ para condiciones de mínima temperatura $(-170 \mathrm{C})$.

Es de notar, en primer lugar, que estipula el alargamiento antes de rotura en lugar de la elongación después de rotura, más usual en la práctica. Ello implica que la experimentación debe incluir medidas extensométricas directas, lo que complica sustancialmente los ensayos, pero suministra, de acuerdo con lo expuesto en (J. Planas et al. 1983), información acerca de un parámetro característico del material, en tanto que la elongación después de rotura depende de factores externos como el tamaño de probeta y las condiciones precisas de ensayo.

En segundo lugar, la especificación KELLOGG indica explicitamente que no es necesario ensayar probetas que contengan defectos, lo que parece indicar que se acepta que la sensibilidad a los defectos es pequeña o que la probabilidad de existencia de defectos de tamaño relevante es escasa.

\subsection{Propuesta en relación con las especificaciones existentes}

En relación al alambre trefilado no se hace ninguna propuesta adicional, pero se resalta la importancia de realizar correctamente la medida del alargamiento bajo carga máxima. Investigaciones desarrolladas en el Departamento de Ciencia de Materiales (J. Planas et al, 1983 ) indicaron que en los aceros de pretensar el perfil de temperaturas a lo largo de la probeta podia influir en los resultados del ensayo de forma notoria, hasta el extremo de aparecer como rechazables materiales verdaderamente aceptables. Se ha establecido que para conseguir resultados válidos era conveniente que los extremos de la probeta estuvieran más fríos que el centro y que en la zona central de la probeta las diferencias de temperatura fueran lo menor posible. La especificación KELLOGG recoge estos resultados y obliga al control del perfil de temperatura en los ensayos de recepción y control.

Aunque no es el único dispositivo posible, un método eficaz y fiable de conseguir los requisitos impuestos, es la utilización de una cámara criostática de tres módulos con control independiente de temperatura como la desarrollada en nuestro Departamento cuyo esquema se ha representado en la -Fig. 3, página siguiente.

En relación con los anclajes o con el comportamiento conjunto del sistema tendón-anclaje, las especificaciones KELLOGG no indican ensayo alguno. Después de la experiencia adquirida al ensayar diversos tipos de anclajes sometidos a distintas solicitaciones (J. Planas et al, 1985) parece razonable realizar una homologación, en condiciones criogénicas, del sistema de pretensado que vaya a ser utilizado.

Esta homologación debe garantizar que el conjunto tendón-anclaje no pueda romper frágilmente bajo las acciones previstas; ello implica que la tensión de rotura del conjunto, a la temperatura deseada, debe ser superior al límite elástico del tendón, a esta misma temperatura. Parece razonable complementar este requisito imponiendo un alargamiento mínimo del conjunto (del $2 \%$, por ejemplo) siguiendo las recomendaciones para temperatura ambiente (FIP, 1981). El valor de este alargamiento a bajas temperaturas es difícil de precisar porque a temperatura ambiente el alargamiento está muy condicionado por el tesado del tendón (que suele ser la condición más restrictiva), mientras que a bajas temperaturas deberá ser función de las deformaciones máximas esperadas en caso de accidente. 


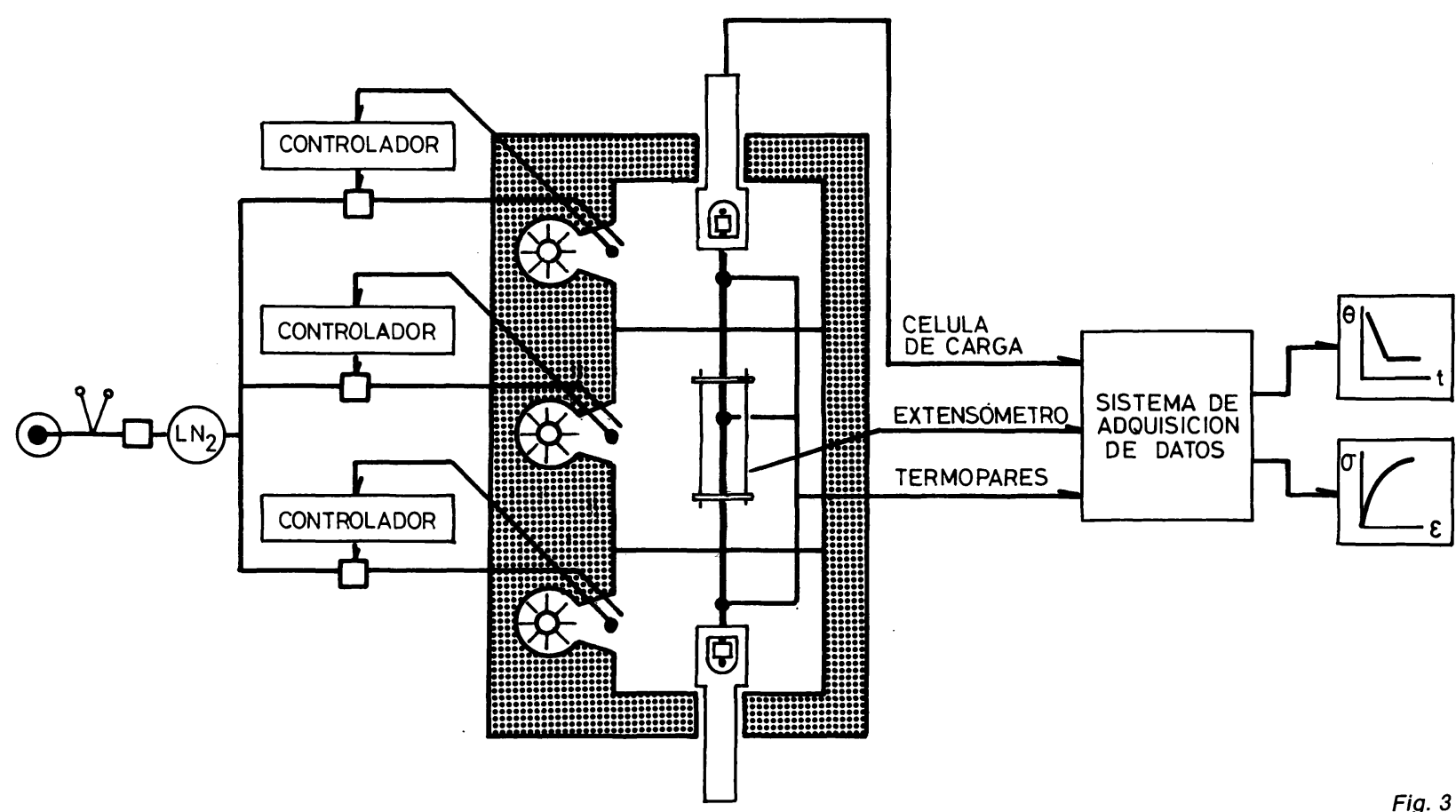

También sería conveniente ensayar el comportamiento, a baja temperatura, de los materiales del anclaje. En particular su tenacidad de fractura para poder estimar el tamaño de los defectos admisibles siguiendo las ideas expuestas en el citado artículo (J. Planas et al, 1985).

\section{ARMADURAS PASIVAS}

\subsection{Comportamiento en condiciones criogénicas}

Los aceros que se utilizan son los tradicionales, al carbono-manganeso, en sus versiones de dureza natural (laminados en caliente) y los endurecidos por deformación en frío. Estos aceros pueden presentar un comportamiento frágil a bajas temperaturas y por este motivo se han desarrollado aceros especiales para hormigón armado en condiciones criogénicas. Estos últimos se consiguen por microaleación y tratamientos térmicos especiales o bien simplemente por aleación. Aqui se analizan sólo los aceros al carbono manganeso que han sido los usados en la construcción del tanque de GNL.

A la temperatura del GNL el límite elástico y la carga de rotura de estos aceros experimentan un notable aumento (al $40 \%$, o más, en todos los casos ensayados). Las restantes propiedades como la deformación en rotura y la sensibilidad a los defectos dependen mucho de la composición del material y al tratamiento térmico del mismo.

La investigación realizada (M. Elices et al, 1986) sobre un acero de armar de dureza natural y otro deformado en frío mostró que el primero mantenía deformaciones bajo carga máxima superiores al $11 \%$ hasta temperaturas de $-180 \mathrm{C}$, en tanto que para el segundo este parámetro caía de $7,6 \%$ a temperatura ambiente a tan sólo el $1,8 \%$ a $180 \mathrm{C}$. A $-165 \mathrm{C}$ se dieron casos de rotura por debajo del límite elástico, con deformaciones totales de sólo el 0,4 \% y fractura iniciada en un mínimo defecto superficial. En este caso tan extremo era obvio que el acero deformado en frío no resultaba adecuado para ser usado en condiciones criogénicas, pero hoy por hoy no podemos concluir que esto sea aplicable a todos los aceros de armar deformados en frío.

La subsiguiente investigación sobre la sensibilidad del acero de dureza natural a los defectos mostró que, a $-165 \mathrm{C}$, era prácticamente insensible a entallas de menos de $0,4 \mathrm{~mm}$ de profundidad y que la carga de rotura superaba siempre el límite elástico para entallas de hasta $2,5 \mathrm{~mm}$ de profundidad (aunque la deformabilidad disminuía drásticamente para entallas de más de $0,5 \mathrm{~mm}$, tal como puede verse en la Fig. 4).

Estos resultados parecian indicar que el acero de dureza natural podría ser usado en condiciones criogénicas, ya que la posibilidad de concurrencia de defectos de más de $0,5 \mathrm{~mm}$ en un número elevado de barras y distantes menos de la longitud de adherencia de la misma sección era muy remota. De hecho el material cumple las correspondientes especificaciones KE. LLOGG que fueron redactadas con posterioridad a la realización de la investigación y que serán comentadas a continuación. Hoy sabemos que este resultado no es aplicable a todos los aceros de armar de dureza natural y que en este material es preciso extremar las medidas de control. 


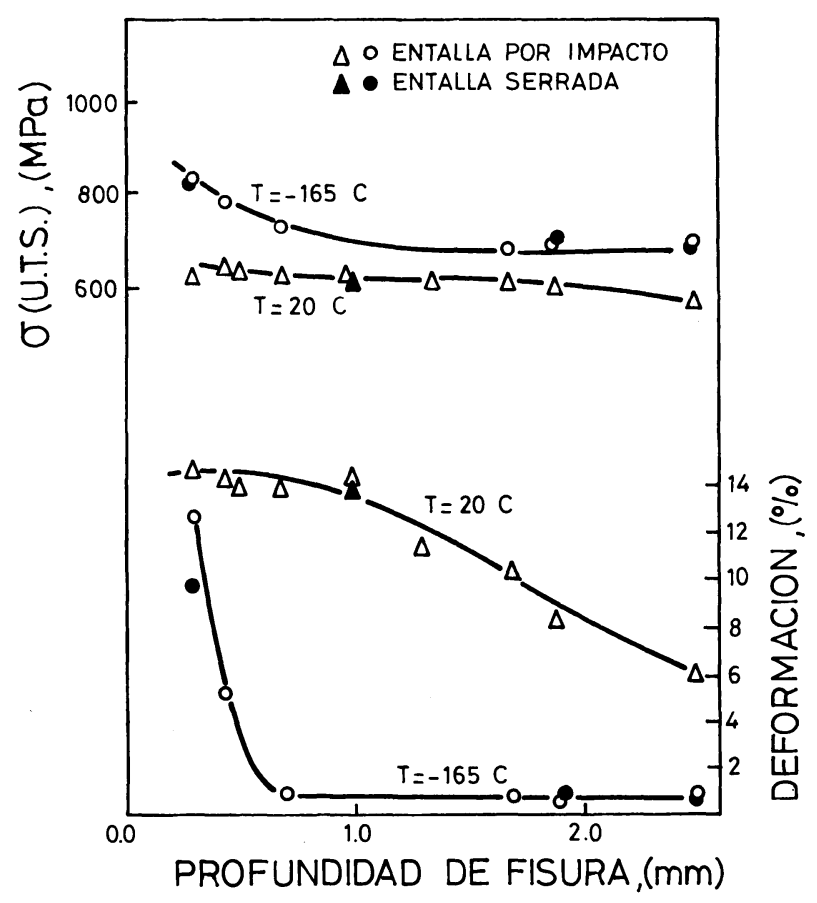

Fig. 4

\subsection{Sobre las prescripciones KELLOGG}

La correspondiente especificación KELLOGG establece que deben realizarse ensayos sobre probetas lisas y sobre probetas con una entalla mecanizada de más de $1 \mathrm{~mm}$ de profundidad, ambos a la misma temperatura de trabajo $(-170 \mathrm{C})$. Establece cuatro condiciones de aceptación que, detalles aparte, son:

1. El límite elástico de probetas no entalladas debe ser superior al requerido para temperatura ambiente en no menos de un $15 \%$.

2. La elongación después de rotura medida sobre 10 diámetros debe superar el $6 \%$ para las probetas lisas.

3. El límite elástico de probetas entalladas debe superar el requerido para temperatura ambiente en probetas lisas.

4. El límite elástico medio de las probetas entalladas no debe ser inferior al $90 \%$ del límite elástico medio de las probetas no entalladas a baja temperatura.

La experiencia acumulada indica que las condiciones más restrictivas son la $2 .^{a}$ y la $4 .^{a}$, que imponen el grado de ductilidad exigido al material.

\subsection{Propuesta en relación con las especificaciones existentes}

Las especificaciones actuales tienden a garantizar una resistencia y una ductilidad mínimas para las armadu- ras pasivas mediante ensayos de tracción. Para tener en cuenta los posibles daños que las armaduras hayan podido sufrir durante el transporte, almacenamiento y colocación en obra, también se comprueban estos requisitos de ductilidad y resistencia con probetas en las que previamente se ha hecho una entalla normalizada de $1 \mathrm{~mm}$ de profundidad. Ambos ensayos (probetas con entalla y sin ella) parecen necesarios y los comentarios que siguen van dirigidos a la forma de realizarlos y al alcance de los resultados.

El ensayo a bajas temperaturas con probetas sin entallar es delicado porque debe registrarse la curva cargaalargamiento, medido sobre una base prefijada y a lo largo de la cual la temperatura debe ser lo más homogénea posible (la diferencia entre dos puntos no debería superar $2^{\circ} \mathrm{C}$ ), por las mismas razones expuestas al comentar las armaduras activas (J. Planas et al, 1983). El punto más cuestionable es el valor exigido para el alargamiento remanente (6\% sobre 10 diámetros). Esta cifra no puede basarse en la experiencia constructiva porque se construye a temperatura ambiente y se supone que los requisitos de ductilidad a temperatura ambiente los cumple. Es preciso justificarlo a partir de las deformaciones máximas esperadas a la temperatura del ensayo y, si éstas son muy inferiores al $6 \%$, es posible que se rechacen partidas de acero perfectamente admisibles para este tipo de estructura.

Un razonamiento análogo puede hacerse para las probetas entalladas. Es posible que la entalla tipificada de $1 \mathrm{~mm}$ de profundidad sea un daño excesivo 0 , por el contrario, que durante la construcción del depósito se puedan producir daños mayores. Sería muy conveniente realizar un estudio de los posibles daños a las armaduras durante el transporte, manejo y almacenamiento. No sólo los golpes pueden ocasionar entallas o mellas, un ambiente agresivo puede formar picaduras y en determinadas condiciones se pueden formar fisuras que pueden ser más peligrosas que entallas de la misma profundidad. El ensayo también es delicado y debe realizarse una cuidadosa medida de las entallas para comprobar su profundidad y radio en el fondo de la entalla. Cuando los resultados del ensayo sean anómalos conviene realizar medidas con un proyector de perfiles $y$, si es preciso, un estudio fractográfico. Por último, la situación de la entalla en barras corrugadas deberá decidirse teniendo en cuenta la simulación de un posible daño real y no buscando el punto donde la concentración de tensiones es más desfavorable.

También sería deseable repetir estos ensayos a varias temperaturas, entre temperatura ambiente y la temperatura de transición, ya que en varias hipótesis de cálculo las armaduras pasivas se supone que están a temperaturas distintas de la del GNL.

Finalmente se recomienda que el nivel de control para las armaduras pasivas sea superior al normal debido 
a la dispersión que se ha observado en estos materiales (M. Elices y G. Sleigh, 1978 y monografía de la FIP, 1982).

AGRADECIMIENTO: Los autores desean expresar su agradecimiento al Dr. Ing. Luis Fort López-Tello por su continuo interés y apoyo durante la realización de este trabajo.

\section{REFERENCIAS}

1. Corres, H., Elices, M. and Planas, J. "Thermal deformation of loaded concrete at Low Temperatures 3 : lightweight concrete". Cement and concrete research. Vol 16, pp 845-852.

2. Elices, M. and Sleigh, G. "Steels for prestressing concrete structures for cryogenic applications". Proceedings $8^{\text {th }}$ FIP Congress, May 1978.

3. Elices, M., Rotasy, F.S. y Faas, W.M., "Cryogenic Behaviour of Materials for Prestressed Concrete. State of Art Report", FIP Ed., Wexham Springs, pp. 111 (1982).

4. Elices, M., "Fracture of Steels for Reinforcing and Prestressing Concrete", in Fracture Mechanics of
Concrete", Sih and Di Tommaso Eds., Martinus Nijhoff Publishers, The Hague, pp. 226-271 (1985).

5. Elices, M., Corres, H. y Planas, J., "Behaviour at Cryogenic Temperatures of Steel for Reinforcement", ACl Journal, Vol. 83, n. 3 3, pp. 405-411 (1986).

6. Elices, M., Planas, J. y Corres, H., "Thermal Deformation of Loaded Concrete at Low Temperatures, 2: Transverse Deformation", Cemente and Concrete Research, Vol. 16 pp. $741-748$ (1986).

7. FIP. "Recommendations for Acceptance and Application of Post-Tensioning Systems" - FIP Report 5.9 (Marzo 1981).

8. Planas, J., Corres, H., Elices, M., Sánchez-Gálvez, V., "Tensile Tests of Steels at low Temperatures, Problems due to Non-Uniformity in Temperature Distribution along the Specimen", Proc. 2nd. Int. Conf. on Cryogenic Concrete, Amsterdam, Vol. III, pp. 10 (1984).

9. Planas, J., Corres, H., Elices, M. y Chueca, R., "Thermal Deformation of Loaded Concrete during Thermal Cycles from $20 \mathrm{C}$ to - 165 C", Cement and Concrete Research, Vol. 14, pp. 639-644 (1984).

10. Planas, J., Corres, H., Elices, M. y Chueca, R. "Comportamiento de sistemas de pretensado a temperaturas criogénicas", Hormigón y Acero, n. ${ }^{0} 155$, pp. 55-70 (1985).

\section{publicación del IETcc / CSIC}

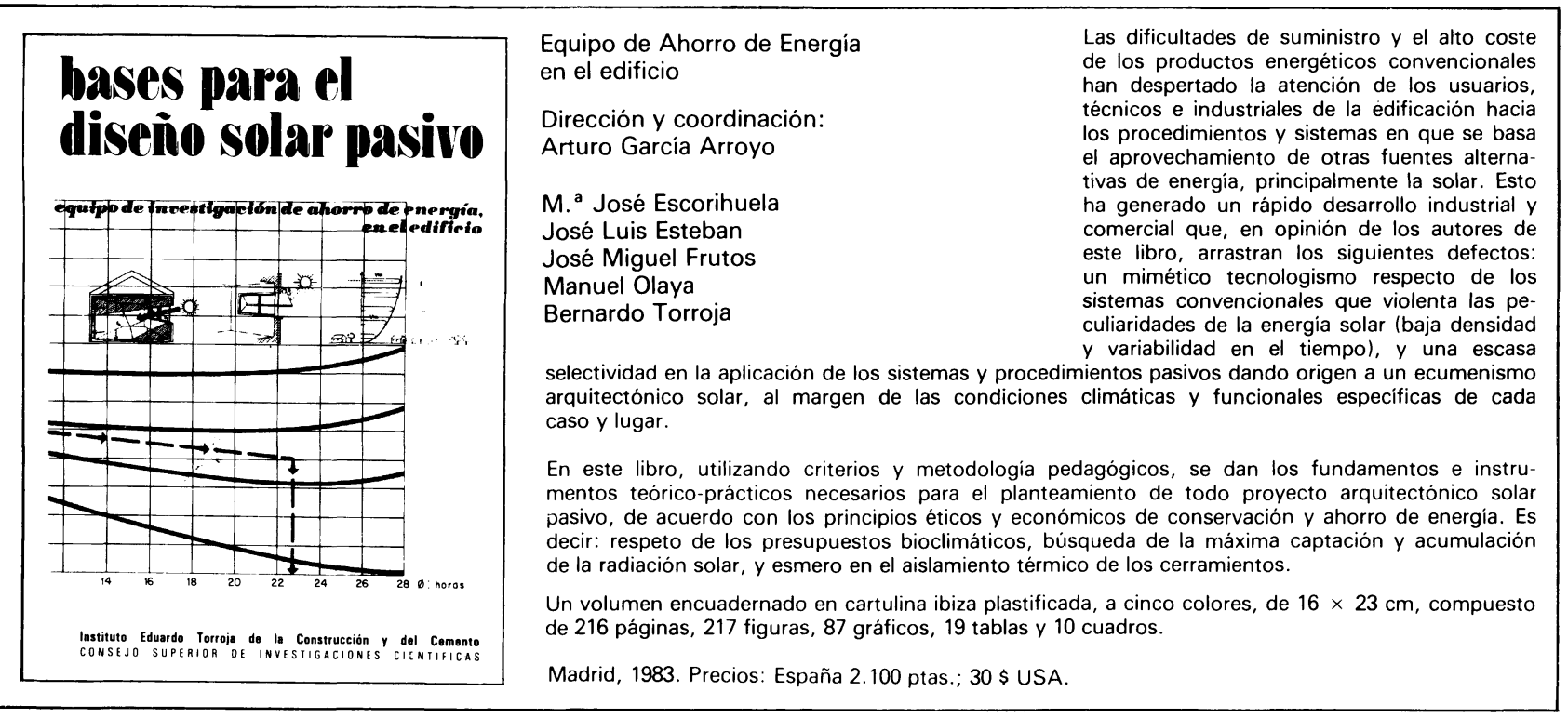

